\title{
Discussion mathématique du modèle de Pauli et Fierz relatif à la catastrophe infrarouge
}

\author{
PHILIPPE BLANCHARD \\ Seminar für theoretische Physik, ETH Zürich
}

Reçu le 5 Juin 1969

\begin{abstract}
In this model we study the interaction of an quantized non relativistic electron with the photon field. We discuss the system for all finite time and we are in the position to construct explicitly the asymptotic states.
\end{abstract}

\section{Introduction}

La catastrophe infrarouge en théorie quantique des champs se rencontre chaque fois que l'on veut décrire des particules de masse nulle. En effet apparaissent alors des états contenant un nombre infini de quanta de si petite énergie que l'énergie totale d'un tel état est cependant finie.

Les travaux de Bloch et Nordsieck [1] (1937) et Pauli et Fierz [2] (1938) constituèrent un progrès essentiel dans la compréhension de ces questions. Ils montrèrent que dans les processus électromagnétiques de diffusion un nombre infini de photons était ordinairement émis.

En étudiant la diffusion de quanta de masse nulle par une source fixe Friedrichs [3] donna une analyse théorique de ces phénomènes. Cette analyse montre qu'une description correcte de telles situations doit se faire à l'aide de représentations des relations canoniques de commutation qui ne sont pas unitairement équivalentes à la représentation de Fock. Le fait d'utiliser en théorie des perturbations cette représentation non appropriée conduit aux divergences infrarouges familières de certaines intégrales [4].

Le modèle de Pauli et Fierz décrit dans une certaine approximation l'interaction d'un électron quantique non relativiste et d'un champ de photons. Dans ce modèle on verra que l'hamiltonien $H$ est un opérateur bien défini et qu'il est possible de lui associer un groupe unitaire à un paramètre qui permet de décrire de façon non ambigue le développement des états dans le temps et de construire les états asymptotiques du système. C'est dans la construction de ces états asymptotiques qu'apparai- 
tront des espaces de Hilbert auxquels seront associées des représentations des relations canoniques de commutation non équivalentes à la représentation de Fock.

La convergence en représentation d'interaction de $\Psi(t)$, qui pour tout $t$ fini est un vecteur de l'espace de Fock, vers $\Psi_{\text {out }}^{\text {in }}$ sera définie à l'aide de la convergence pour $|t| \rightarrow+\infty$ des valeurs moyennes $\langle\Psi(t), A \Psi(t)\rangle$ des opérateurs $A$ d'une certaine algèbre.

\section{Le modèle de Pauli-Fierz}

On considère une particule chargée de masse $m$, de charge $e$ et de distribution de charge $e \varrho(|\xi|)$ où $\varrho$ est une fonction réelle, sphériquement symétrique, obéissant à une condition de normalisation

$$
\int \varrho(|\xi|) d^{3} \xi=1, \quad \varrho \in \mathscr{S}\left(\mathbb{R}^{3}\right) .
$$

La transformée de Fourier de $\varrho$, $\varrho$, est un facteur de forme qui évitera l'apparition d'une catastrophe ultraviolette.

Cette particule est soumise à l'action d'un potentiel extérieur $V(\boldsymbol{x})$ et est couplée avec le champ de rayonnement.

Posant $\hbar=c=1$ l'hamiltonien s'écrit dans l'approximation non relativiste

$$
H=\frac{1}{2 m}\left(\boldsymbol{p}-e \boldsymbol{A}_{0}\right)^{2}+\frac{1}{2} \int: \boldsymbol{E}_{t r}^{2}+\mathbf{B}^{2}: d^{3} x+V(\boldsymbol{x})
$$

avec

$$
\boldsymbol{A}_{0}(\boldsymbol{x})=\int \boldsymbol{A}_{t r}(\boldsymbol{x}+\xi) \varrho(\xi) d^{3} \xi .
$$

$\boldsymbol{A}_{t r}(\boldsymbol{x})$ est le potentiel vecteur transversal défini par sa décomposition de Fourier

$$
\begin{aligned}
A_{t r}(x) & =\frac{1}{(2 \pi)^{3 / 2}} \int \frac{d^{3} k}{\sqrt{2 k}} \sum_{s=1}^{2} \boldsymbol{e}_{s}(\boldsymbol{k})\left\{a_{s}(\boldsymbol{k}) e^{i(\boldsymbol{k}, \boldsymbol{x})}+a_{s}^{*}(\boldsymbol{k}) e^{-i(\boldsymbol{k}, \boldsymbol{x})}\right\} \\
k & =|\boldsymbol{k}|
\end{aligned}
$$

On a adopté la jauge de radiation de telle sorte que seul le potentiel vecteur est utilisé pour décrire le champ de rayonnement. Les variables dynamiques du problème sont $\boldsymbol{x}, \boldsymbol{p}, a_{s}(\boldsymbol{k}), \mathrm{a}_{s}^{*}(\boldsymbol{k})$ avec les règles de commutation habituelle:

$$
\left[a_{s}(\boldsymbol{k}), a_{s^{\prime}}^{*}\left(\boldsymbol{k}^{\prime}\right)\right]=\delta_{s s^{\prime}} \delta\left(\boldsymbol{k}-\boldsymbol{k}^{\prime}\right) .
$$

Les deux premières variables commutent avec les deux autres. L'espace de Hilbert que l'on considère est le produit tensoriel de l'espace d'im- 
pulsion de l'électron par l'espace de Fock des photons

$$
\mathscr{H}=L^{2}\left(\mathbb{R}^{3}\right) \otimes \mathscr{F} .
$$

Du fait de la transversalité on a aussi les proprietés suivantes

$$
\begin{aligned}
\left(\boldsymbol{k}, \boldsymbol{e}_{s}(\boldsymbol{k})\right) & =0, \\
\left(\boldsymbol{e}_{s}(\boldsymbol{k}), \boldsymbol{e}_{s^{\prime}}(\boldsymbol{k})\right) & =\delta_{s s^{\prime}}
\end{aligned}
$$

On se propose de traiter ce modèle dans l'approximation dipolaire c'est à dire de remplacer $\boldsymbol{A}_{0}(\boldsymbol{x})$ par $\boldsymbol{A}_{0}(\mathbf{0})$. Ceci revient à négliger la conservation de l'impulsion. D'autre part on ne tient pas compte non plus du terme en $\boldsymbol{A}_{0}^{2}$. La justification de ces approximations est discutée dans [2]. On considère donc l'hamiltonien suivant:

$$
\begin{aligned}
H= & \frac{\boldsymbol{p}^{2}}{2 m}+\sum_{s=1}^{2} \int d^{3} k k a_{s}^{*}(\boldsymbol{k}) a_{s}(\boldsymbol{k})+V(\boldsymbol{x}) \\
& -\frac{e}{m} \sum_{s=1}^{2} \int \frac{d^{3} k}{\sqrt{2 k}} \tilde{\varrho}(\boldsymbol{k})\left(\boldsymbol{p}, \boldsymbol{e}_{s}(\boldsymbol{k})\right)\left[a_{s}(\boldsymbol{k})+a_{s}^{*}(\boldsymbol{k})\right], \\
\varrho(\boldsymbol{k})= & \int \varrho(\xi) e^{i(\boldsymbol{k}, \xi)} d^{3} \xi, \quad \tilde{\varrho}(0)=1, \quad \varrho \in \mathscr{S}\left(\mathbb{R}^{3}\right) .
\end{aligned}
$$

On définit $H^{\prime}$ par

$$
H=H_{0}^{\mathrm{el}}+H_{0}^{\mathrm{ray}}+V+H_{I}=H^{\prime}+V
$$

avec

$$
\begin{aligned}
H_{0}^{\mathrm{el}} & =\frac{\boldsymbol{p}^{2}}{2 m} \\
H_{0}^{\mathrm{ray}} & =\sum_{s=1}^{2} \int d^{3} k k a_{s}^{*}(\boldsymbol{k}) a_{s}(\boldsymbol{k}), \\
H_{I} & =-\frac{e}{m} \sum^{2} \int \frac{d^{3} k}{\sqrt{2 k}} \tilde{\varrho}(\boldsymbol{k})\left(\boldsymbol{p}, \boldsymbol{e}_{s}(\boldsymbol{k})\right)\left[a_{s}(\boldsymbol{k})+a_{s}^{*}(\boldsymbol{k})\right] .
\end{aligned}
$$

Notre but est de définir les états asymptotiques du système associé à cet hamiltonien.

Nous l'atteindrons en deux temps:

1. discussion du système associé à $H^{\prime}=H_{0}^{\text {el }}+H_{0}^{\text {ray }}+H_{I}$ et étude de son comportement asymptotique pour $|t| \rightarrow \infty$.

2. Propriétés du système associé à $H=H^{\prime}+V$ et démonstration de l'existence des limites fortes suivantes sur $\mathscr{H}$ :

$$
\Omega^{ \pm}=S-\lim _{t \rightarrow \pm \infty} e^{i H t} e^{-i H^{\prime} t} .
$$




\section{Le système «libre» $H^{\prime}=H_{0}^{\text {el }}+H_{0}+H_{I}$}

$\mathscr{H}$ peut s'écrire $\mathscr{H}=\int_{\oplus} \mathscr{F}(\boldsymbol{p}) d^{3} p$. Ceci revient à dire que les vecteurs $\Phi \in \mathscr{H}$ sont définis comme des fonctions $\boldsymbol{p} \rightarrow \Phi(\boldsymbol{p})$ à valeurs dans $\mathscr{F}$. Ces fonctions sont faiblement mesurables et de carré intégrable. On entend par là que:

1) La fonction $(\Phi(p), \Psi)$ dépend mesurablement de $p \forall \Psi \in \mathscr{H}$,

2) le champ de vecteurs $\Phi(\boldsymbol{p})$ est de carré intégrable:

$$
\int\|\Phi(\boldsymbol{p})\|^{2} d^{3} p<\infty .
$$

Du fait de l'approximation dipolaire $\boldsymbol{p}$ est une intégrale du mouvement $\left[\boldsymbol{p}, H^{\prime}\right]=0$ et $e^{-i H^{\prime} t}$ est une fonction $\boldsymbol{p} \rightarrow e^{-i H^{\prime} t}(\boldsymbol{p})$ à valeur opérateur dans $\mathscr{F}$. On verra que pour tout $t$ fini c'est un opérateur unitaire dans $\mathscr{F}$.

Ecrivons formellement en représentation d'interaction

$$
e^{-i H^{\prime} t}(\boldsymbol{p})=e^{-i H_{0}^{\mathrm{ray}} t} e^{\frac{-i \boldsymbol{p}^{2}}{2 m}} U(\boldsymbol{p}, t)
$$

$U(\boldsymbol{p}, t)$ verifiant l'équation

$$
\left\{\begin{aligned}
i \frac{\partial}{\partial t} U(\boldsymbol{p}, t) & =H_{I}(t) U(\boldsymbol{p}, t), \\
U(\boldsymbol{p}, 0) & =\mathbb{1}
\end{aligned}\right\}
$$

avec

$$
\begin{aligned}
& H_{I}(t)=e^{i H_{0}^{\mathrm{ray}} t} H_{I} e^{-i H_{0}^{\mathrm{ray}} t}, \\
& H_{I}(t)=-\frac{e}{m} \sum_{s=1}^{2} \int \frac{d^{3} k}{\sqrt{2 k}} \tilde{\varrho}(\boldsymbol{k})\left(\boldsymbol{p}, \boldsymbol{e}_{s}(\boldsymbol{k})\right)\left[a_{s}(\boldsymbol{k}) e^{-i k t}+a_{s}^{*}(\boldsymbol{k}) e^{i k t}\right] .
\end{aligned}
$$

On peut intégrer l'Eq. (1) car le commutateur $\left[H_{I}(t), H_{I}\left(t^{\prime}\right)\right]$ est un nombre sur $\mathscr{F}$. (Méthode de Magnus [5].) En effet rappelons que si

$$
[A,[A, B]]=[B,[A, B]]=0,
$$

on a l'identité

$$
e^{A+B}=e^{A} e^{B} e^{-\frac{1}{2}[A, B]}
$$

or

$$
\left[H_{I}(t), H_{I}\left(t^{\prime}\right)\right]=-\frac{2}{3} i \frac{e^{2} \boldsymbol{p}^{2}}{m^{2}} \int d^{3} k \frac{[\varrho(k)]^{2}}{k} \sin k\left(t-t^{\prime}\right) .
$$

On a alors:

$$
U(\boldsymbol{p}, t)=\mathrm{e}^{-i \int_{0}^{t} H_{I}\left(t^{\prime}\right)-\frac{1}{2} \int_{0}^{t} d t^{\prime} \int_{0}^{\prime} d t^{\prime \prime}\left[H_{I}\left(t^{\prime}\right), H_{I}\left(t^{\prime \prime}\right)\right]}
$$


or

$$
-\frac{1}{2} \int_{0}^{t} d t^{\prime} \int_{0}^{t^{\prime}} d t^{\prime \prime}\left[H_{I}\left(t^{\prime}\right), H_{I}\left(t^{\prime \prime}\right)\right]=-\frac{i e^{2} p^{2} t}{3 m^{2}} \int \frac{\varrho^{2}(k)}{k^{2}}\left[1-\frac{\sin k t}{k t}\right] d^{3} k .
$$

On trouve ainsi

$$
e^{-i H^{\prime} t}(\boldsymbol{p})=e^{-i H_{0}^{\mathrm{ray}} t} e^{-i \frac{\boldsymbol{p}^{2} t}{2 m(t)}} e^{-i(\boldsymbol{p}, \boldsymbol{A}(t))},
$$

$m(t)$ et $\boldsymbol{A}(t)$ étant respectivement définis par

$$
\left.\begin{array}{l}
\text { (2a) } \frac{1}{m(t)}=\frac{1}{m}-\frac{2}{3} \frac{e^{2}}{m^{2}} \int d^{3} k \frac{[\tilde{\varrho}(k)]^{2}}{k^{2}}\left(1-\frac{\sin k t}{k t}\right), \\
\text { (2b) } \boldsymbol{A}(t)=-\frac{e}{m} \sum_{s=1}^{2} \int d^{3} k \frac{\tilde{\varrho}(k)}{k \sqrt{2 k}} \boldsymbol{e}_{s}(\boldsymbol{k})\left[a_{s}(\boldsymbol{k}) u(k, t)+a_{s}^{*}(\boldsymbol{k}) \overline{u(k, t)}\right], \\
\text { (2c) } u(k, t)=i\left(e^{-i k t}-1\right) .
\end{array}\right\}
$$

Pour tout $t$ fini $\boldsymbol{A}(t)$ est un opérateur bien défini de l'espace de Fock, on a en effet $\forall|t|<\infty$

$$
\frac{\tilde{\varrho}(k)}{k \sqrt{2 k}} u(k, t) \in L^{2}\left(\mathbb{R}^{3}\right)
$$

Definissons $N(t)$ par

$$
\begin{aligned}
& N^{2}(t) \equiv \frac{e^{2}}{m^{2}} \int d^{3} k \frac{[\tilde{\varrho}(k)]^{2}}{2 k^{3}}|u(k, t)|^{2}, \\
& N^{2}(t)=\frac{8 \pi e^{2}}{m^{2}} \int_{0}^{\infty} d k \frac{[\tilde{\varrho}(k)]^{2}}{k} \sin ^{2} \frac{k t}{2}<\infty .
\end{aligned}
$$

Il en résulte que $e^{-i H^{\prime} t}$ est lui aussi bien défini. C'est un groupe unitaire continu à un paramètre. $H^{\prime}$ est défini comme le générateur infinitésimal de ce groupe, est self adjoint et coincide avec l'expression formelle de $H^{\prime}$ pour un ensemble d'états denses dans l'espace de Hilbert $\mathscr{H}$ (cf IV b) pour une définition précise de ces états).

\section{Catastrophe infrarouge}

Pour $t$ fini la catastrophe infrarouge ne se manifeste pas. Tout se passe comme si les photons n'avaient pas une masse nulle. C'est pour $t \rightarrow \pm \infty$ que la catastrophe infrarouge apparait. Quand $|t| \rightarrow \infty m(t)$ converge vers la masse renormalisée $m(\infty)$ mais $N^{2}(t)$ diverge loga- 
rithmiquement

$$
N^{2}(t) \approx \log |t|
$$

En effet considérons l'expression (3)

$$
\begin{aligned}
\int_{0}^{\infty} d k \frac{\tilde{\varrho}^{2}(k)}{k} \sin ^{2} \frac{k t}{2}= & \int_{0}^{1} d k \frac{\varrho^{2}(k)-\tilde{\varrho}^{2}(0)}{k}(1-\cos k t) \\
& +\varrho^{2}(0) \int_{0}^{1} d k \frac{1-\cos k t}{k}+\int_{1}^{\infty} d k \frac{\tilde{\varrho}^{2}(k)}{k} \sin ^{2} \frac{k t}{2} .
\end{aligned}
$$

La première et la troisième intégrale du second membre convergent vers des quantités finies quand $t \rightarrow+\infty$. Quant à la seconde posant $k t=x$ elle s'écrit compte tenu des propriétés du cosinus intégral:

$$
\tilde{\varrho}^{2}(0) \int_{0}^{t} \frac{d x}{x}(1-\cos x)=\tilde{\varrho}^{2}(0)\left[\varepsilon+\log t+\int_{t}^{\infty} \frac{\cos x}{x} d x\right],
$$

$\varepsilon$ étant la constante d'Euler.

On se propose maintenant de discuter les propriétés asymptotiques dy système associé à $H^{\prime}$ pour $t \rightarrow \pm \infty$. On remarque tout d'abord que quand $t \rightarrow \pm \infty$

$$
\begin{aligned}
e^{-i \frac{\boldsymbol{p}^{2} t}{2 m(t)}} & =e^{-i \frac{\boldsymbol{p}^{2} t}{2 m(\infty)} \pm i \boldsymbol{p}^{2} \lambda+o(|t|)} \\
\lambda & =-\frac{4 \pi e^{2}}{m^{2}} \int_{0}^{\infty} \frac{\sin \alpha}{\alpha} d \alpha=-\frac{2 \pi^{2} e^{2}}{m^{2}} .
\end{aligned}
$$

D'autre part on s'attend à ce que $e^{-i(\boldsymbol{p}, \boldsymbol{A}(t))}$ converge «dans un certain sens» vers $e^{-i(\boldsymbol{p}, \boldsymbol{A}(\infty))}, \boldsymbol{A}(\infty)$ s'obtenant à partir de $\boldsymbol{A}(t)$ en remplaçant $u(k, t)=i\left(e^{-i k t}-1\right)$ par $-i$. Dans une théorie de la diffusion simple on trouverait alors que

$$
\begin{aligned}
\Omega^{ \pm *} & =\lim _{t \rightarrow \pm \infty} e^{i\left(\frac{\boldsymbol{p}^{2}}{2 m(\infty)}+H_{0}^{\mathrm{ray}}\right) t} e^{-i H^{\prime} t} \\
& =e^{ \pm i \boldsymbol{p}^{2} \lambda} e^{-i(\boldsymbol{p}, \boldsymbol{A}(\infty))}
\end{aligned}
$$

La situation est ici plus compliquée.

Afin de simplifier les notations posons

$$
\begin{aligned}
U(f) & =e^{-i\left[a(f)+a^{*}(f)\right]}, \\
a(f) & =\int d^{3} k f(\boldsymbol{k}) a(\boldsymbol{k}) \quad f \in L^{2}\left(\mathbb{R}^{3}\right) .
\end{aligned}
$$


(Dans tout ce qui suit on ne tient plus compte de la polarisation.) Les propriétés essentielles de $U(f)$ peuvent se résumer dans le lemme ci-après:

Lemme. a) Pour $f \in L^{2}\left(\mathbb{R}^{3}\right), U(f)$ est unitaire et fortement continu par rapport à $f$.

b) Si $\|f\|_{2} \rightarrow \infty, U(f)$ tend faiblement vers zéro sur $\mathscr{F}$.

c) Soit $L^{2}\left(\mathbb{R}^{3}\right)=N_{1} \oplus N_{2}\left(N_{2}=N_{1}^{\perp}\right)$. On a alors $\mathscr{F}\left(L_{2}\left(\mathbb{R}^{3}\right)\right)=\mathscr{F}\left(N_{1}\right)$ $\otimes \mathscr{F}\left(N_{2}\right), \mathscr{F}(H)$ étant l'espace de Fock construit sur $H$.

$$
U(f)=U\left(f_{1}\right) \otimes U\left(f_{2}\right) .
$$

$f_{i}$ étant la projection de $f$ sur $N_{i}, i=1,2$.

Démonstration. a) Soit $\mathscr{F}_{0}$ le sous ensemble de l'espace de Fock constitué par les vecteurs qui n'ont qu'un nombre fini de composantes différentes de zéro. $\mathscr{F}_{0}$ est dense dans $\mathscr{F}$. De plus les vecteurs de $\mathscr{F}_{0}$ sont des vecteurs analytiques [16] de $a(f)+a^{*}(f)$. Comme $U(f)$ est unitaire et que (pour des fonctions $f$ réelles)

$$
U\left(f+f^{\prime}\right)=U(f) U\left(f^{\prime}\right)
$$

il est suffisant de prouver la continuité à l'origine. $\forall \Phi \in \mathscr{F}_{0}$ on a

$$
\left\|\left(a(f)+a^{*}(f)\right)^{k} \Phi\right\|_{\mathscr{F}} \leqq\|f\|_{2}\|\sqrt{(N+1) \ldots(N+k)} \Phi\|_{\mathscr{F}}
$$

où $N$ est l'opérateur nombre de particules.

Il est alors clair que

$$
\|\Phi-U(f) \Phi\| \leqq C(\Phi)\|f\|_{2} .
$$

$C(\Phi)$ étant une constante. Cette dernière inégalité entraine la propriété de continuité annoncée.

b) Il est facile de voir que sur $\widetilde{\mathscr{F}}_{0}$ on a pour $g_{i}, h_{i} \in L^{2}\left(\mathbb{R}^{3}\right)$

$$
\begin{aligned}
\left\langle\left\langle\prod_{i=1}^{N} a^{*}\left(g_{i}\right) \Omega, U(f) \prod_{i=1}^{M} a^{*}\left(h_{i}\right) \Omega\right\rangle\right. & \\
& \leqq \text { const. } e^{-\frac{1}{2}\|f\|_{2}^{2}}\left(1+\|f\|_{2}\right)^{N+M} .
\end{aligned}
$$

Il en résulte bien que $U(f)$ tend faiblement vers zéro quand $\|f\|_{2} \rightarrow \infty$,

c) est une conséquence immédiate des relations canoniques de commutation.

Or $e^{-i(\boldsymbol{p}, \boldsymbol{A}(t))}$ est un opérateur de ce type. Posons

avec

$$
\begin{aligned}
e^{-i(\boldsymbol{p}, \boldsymbol{A}(t))} & =U\left(f_{t}\right), \\
f_{t}(\boldsymbol{k}) & =g(\boldsymbol{k})\left(e^{-i k t}-1\right)
\end{aligned}
$$

$$
k^{3 / 2} g(\boldsymbol{k}) \in \mathscr{S}\left(\mathbb{R}^{3}\right) .
$$


On a vu que $f_{t} \in L^{2}\left(\mathbb{R}^{3}\right)$ pour tout $t$ fini; $g(k)$ est une fonction de $L^{1}\left(\mathbb{R}^{3}\right)$ qui n'est pas de carré intégrable. On sait aussi que $\left\|f_{t}\right\|_{2} \rightarrow \infty$ quand $|t| \rightarrow \infty$ ce qui a pour conséquence d'après le lemme b) que

$$
w-\lim _{|\boldsymbol{t}| \rightarrow \infty} e^{-i(\boldsymbol{p}, \boldsymbol{A}(t))}=0 \quad \text { sur } \mathscr{F} .
$$

Néanmoins nous allons définir une limite unitaire de $U\left(f_{t}\right)$ pour $t \rightarrow \pm \infty$. Pour ce faire nous allons utiliser la théorie des produits tensoriels infinis d'espaces de Hilbert de von Neumann $[8,9]$. Dans ce but nous décomposons $L^{2}\left(\mathbb{R}^{3}\right)$ de la façon suivante

$$
\text { (A) } \quad L^{2}\left(\mathbb{R}^{3}\right)=\bigoplus_{v=1}^{\infty} \mathscr{H}_{v}
$$

de telle sorte que $f_{t}^{v}$ ait une limite $f^{v}$ dans $\mathscr{H}_{v}$

(B) $\left\|f_{t}^{v}-f^{v}\right\|_{2} \rightarrow 0$

quand $|t| \rightarrow \infty$ dans chaque $\mathscr{H}_{v}, f_{t}^{v}$ étant la projection de $f_{t}$ sur $\mathscr{H}_{v}$. L'espace de Fock s'écrit alors

$$
\mathscr{F}=\bigotimes_{v=1}^{\infty}\left\{\Omega_{v}\right\} \mathscr{F}_{v} .
$$

C'est le produit incomplet (IDPS dans la terminologie de von Neumann) associé à la classe d'équivalence du vide $\left\{\Omega_{v}\right\}, \Omega_{v}$ étant le vide de l'espace de Fock $\mathscr{F}_{v}$ construit sur $\mathscr{H}_{v}$.

Compte tenu de cette décomposition de $L^{2}\left(\mathbb{R}^{3}\right) U\left(f_{t}\right)$ s'écrit

$$
U\left(f_{t}\right)=\bigotimes_{v=1}^{\infty} U\left(f_{t}^{v}\right) .
$$

Pour tout temps $t$ fini c'est un opérateur unitaire dans l'espace $\bigotimes_{v=1}^{\infty} \mathscr{F}_{v}$. De plus le sous espace $\mathscr{F}$ est invariant pour $U\left(\mathrm{f}_{t}\right)$. Le fait que $\left\|f_{t}^{v}-f^{v}\right\|_{2} \rightarrow 0$ quand $|t| \rightarrow \infty$ a pour conséquence d'après le lemme a) que

$$
s-\lim _{|t| \rightarrow \infty} U\left(f_{t}^{v}\right)=U\left(f^{v}\right) \quad \text { sur } \mathscr{H}_{v} .
$$

Ceci nous conduit à définir

$$
U(\infty) \equiv \lim _{|t| \rightarrow \infty} U\left(f_{t}\right) \equiv \bigotimes_{v=1}^{\infty} U\left(f^{v}\right) .
$$

C'est un opérateur unitaire sur l'espace produit complet $\bigotimes_{v=1}^{\infty} \mathscr{F}_{v}$. Cet opérateur ne laisse plus le sous espace $\mathscr{F}$ invariant mais l'applique au contraire sur un autre IDPS $\mathscr{H}(\boldsymbol{p})$

$$
\mathscr{H}(\boldsymbol{p})=\bigotimes_{v=1}^{\infty}\left\{U\left(f_{v}\right) \Omega_{v}\right\} \mathscr{F}_{v} .
$$


Néaumoins la convergence de $U\left(f_{t}\right)$ vers $U(\infty)$ peut s'exprimer à l'aide de la convergence des valeurs moyennes $\left\langle U\left(f_{t}\right) \Psi, B U\left(f_{t}\right) \Psi\right\rangle$ des opérateurs d'une certaine algèbre.

Soit $B_{N} \in \mathscr{L}\left(\bigotimes_{v=1}^{N} \mathscr{F}_{v}\right), \mathscr{L}\left(\bigotimes_{v=1}^{N} \mathscr{F}_{v}\right)$ étant la $C^{*}$-algèbre des opérateurs linéaires continus dans $\bigotimes_{v=1}^{N} \mathscr{F}_{v}$. L'opérateur

$$
B^{\prime}=B_{N} \otimes \mathbb{1}_{N+1} \otimes \mathbb{1}_{N+2} \otimes \cdots=B_{N} \otimes \mathbb{1}
$$

est un opérateur borné sur $\bigotimes_{\nu=1}^{\infty} \mathscr{F}_{v}$ qui laisse invariant chaque IDPS. Soient $B$ et $\hat{B}$ les restrictions de $B^{\prime}$ à $\mathscr{F}$ et $\mathscr{H}(\boldsymbol{p})$. On a alors

$$
w-\lim _{|t| \rightarrow \infty} U\left(f_{t}\right)^{-1} B U\left(f_{t}\right)=U(\infty)^{-1} \hat{B} U(\infty) .
$$

Démonstration. Il suffit de considérer les vecteurs $-C_{0}$ tels que $\left\|\Psi_{v}\right\|=1 \forall v$. On a alors

$$
\begin{aligned}
\left\langle U\left(f_{t}\right) \Psi, B U\left(f_{t}\right) \Psi\right\rangle & \\
& =\prod_{v=1}^{N}\left\langle U\left(f_{t}^{v}\right) \Psi_{v}, B_{v} U\left(f_{t}^{v}\right) \Psi_{v}\right\rangle \prod_{v=N+1}^{\infty}\left\langle U\left(f_{t}^{v}\right) \Psi_{v}, U\left(f_{t}^{v}\right) \Psi_{v}\right\rangle \\
& =\prod_{v=1}^{N}\left\langle U\left(f_{t}^{v}\right) \Psi_{v}, B_{v} U\left(f_{t}^{v}\right) \Psi_{v}\right\rangle
\end{aligned}
$$

le second facteur étant egal à 1 et il résulte clairement des propriétés de $U\left(f_{t}^{v}\right)$ que la limite pour $|t| \rightarrow \infty$ de telles expressions existe ce qui entraine la propriété annoncée. Il est aussi évident que l'adhérence normique de l'algèbre des opérateurs du type $B^{\prime}$ possède aussi cette propriété.

En ce qui concernela reqrésentation des relations canoniques de commutation la situation se présente de la manière suivante: supposons de plus que les $\mathscr{H}_{v}$ sont invariants par rapport à la conjugaison complexe dans $L^{2}\left(\mathbb{R}^{3}\right)$. A chaque $\mathscr{F}_{v}$ est associée une représentation de Fock des opérateurs de Weyl [11]

$$
\begin{aligned}
U\left(h_{v}\right) & =e^{i\left[a\left(h_{v}\right)+a^{*}\left(h_{v}\right)\right]}, \\
V\left(h_{v}\right) & =e^{\left[a\left(h_{v}\right)-a^{*}(v)\right]}, \\
h_{v} \in \mathscr{H}_{v}, & h_{v}=\bar{h}_{v}, \\
U\left(h_{v}+g_{v}\right) & =U\left(h_{v}\right) U\left(g_{v}\right), \\
V\left(h_{v}+g_{v}\right) & =V\left(h_{v}\right) V\left(g_{v}\right), \\
U\left(h_{v}\right) V\left(g_{v}\right) & =e^{-2 i\left(h_{v}, g_{v}\right)} V\left(g_{v}\right) U\left(h_{v}\right) .
\end{aligned}
$$


La représentation produit

$$
\begin{aligned}
& U^{\prime}(h)=\bigotimes_{v=1}^{\infty} U\left(h_{v}\right), \\
& V^{\prime}(h)=\bigotimes_{v=1}^{\infty} V\left(h_{v}\right), \\
& h \in L^{2}\left(\mathbb{R}^{3}\right), \quad h=\bar{h}
\end{aligned}
$$

est définie $\operatorname{sur} \bigotimes_{v=1}^{\infty} \mathscr{F}_{v}$. On sait qu'elle laisse $\mathscr{F}$ invariant et que sa restriction à $\mathscr{F}$ est encore une représentation de Fock. Il est facile de voir que $\mathscr{H}(\boldsymbol{p})$ sera lui aussi laissé invariant si et seulement si

$$
\text { (C) } \quad \sum_{v}\left|\left(f^{v}, h_{v}\right)\right|<\infty \text {. }
$$

Soient encore $U(h)$ et $\hat{U}(h)$ les restrictions de $U^{\prime}(h)$ à $\mathscr{F}$ et $\mathscr{H}(\boldsymbol{p})$.

D'après un theorème de Klauder Mac Kenna et Woods $[10,11]$ on sait que la représentation $\hat{U}(f) \hat{V}(f)$ est irréductible et équivalente à la représentation de Fock $U(f) V(f)$ si et seulement si les vecteurs $-C_{0}\left\{\Omega_{v}\right\}$ et $\left\{U\left(f^{v}\right) \Omega_{v}\right\}$ sont faiblement équivalents c'est à dire si

$$
\sum_{v}||\left(\Omega_{v}, U\left(\mathrm{f}^{v}\right) \Omega_{v}\right)|-1|<\infty .
$$

Indiquons maintenant comment la décomposition (A) se laisse réaliser de manière concrète et ceci de façon à ce que les conditions $(\mathrm{B}),(\mathrm{C})$ soient satisfaites

a) on peut introduire une base orthonormale dans $L^{2}\left(\mathbb{R}^{3}\right)$ (fonctions d'Hermite par exemple). $\mathscr{H}_{v}$ est alors de dimension 1 et la condition (B) s'exprime par une condition de régularité au voisinage de $k=0$. Plus précisément il faut que

$$
\int \frac{\varrho(k)}{k^{3 / 2}} u_{v}(\boldsymbol{k}) d^{3} k<\infty
$$

les $u_{v}(\boldsymbol{k})$ étant les vecteurs de base. La condition (C) sera surement vérifiée si les vecteurs de base sont pris dans $\mathscr{S}\left(\mathbb{R}^{3}\right)$;

b) on peut aussi envisager un recouvrement de $\mathbb{R}^{3}$

$$
\mathbb{R}^{3}=\bigcup_{v=1}^{\infty} C_{v}
$$

les $C_{v}$ étant des compacts disjoints tels que tout compact de $\mathbb{R}^{3}$ soit contenu dans une réunion finie des $C_{v}$. 
Utilisant le lemme de Riemann-Lebesque il est alors facile de voir que dans l'espace de configuration

$$
\left\|f_{t}^{v}-g^{v}\right\|_{2} \rightarrow 0
$$

où $g^{v}$ est la restriction au compact $C_{v}$ de la transformée de Fourier de $\tilde{\varrho}(k) / k^{3 / 2}$. Les observables $B$ à l'aide desquelles on définit la convergence de $U\left(f_{t}\right)$ vers $U(\infty)$ sont dans ce cas les observables locales.

$U(\infty)^{-1}$ applique $\mathscr{H}(\boldsymbol{p})$ sur $\mathscr{F}$. C'est aussi un isomorphisme des algèbres d'opérateurs associées à $\mathscr{F}$ et $\mathscr{H}(\boldsymbol{p})$. On a une représentation

$$
\hat{a}(h)=a(h)-i(g, h)
$$

des relations canoniques de commutation.

Dans ce formalisme on peut aussi aisément discuter l'existence de la limite faible d'expressions du type

$$
U\left(f_{t}\right)^{-1} B U\left(f_{t}\right)
$$

pour certaines classes d'opérateurs non bornés $B$. On peut par exemple s'intéresser aux opérateurs définis par

$$
\int r(\boldsymbol{k}) a^{*}(\boldsymbol{k}) a(\boldsymbol{k}) d^{3} k .
$$

On s'aperçoit qu'on peut donner un sens à cette limite pour l'opérateur énergie, pour l'opérateur impulsion. Par contre comme on doit s'y attendre, elle n'a pas de sens pour l'opérateur nombre de photons. Un calcul très simple montre en effet que, pour que ces limites faibles existent sur un ensemble dense de vecteurs, il suffit que la fonction $r(\boldsymbol{k})$ soit telle que $r g \in L^{2}\left(\mathbb{R}^{3}\right)$ et que $r g^{2} \in L^{1}\left(\mathbb{R}^{3}\right)$.

On a vu aussi que $\mathscr{H}(\boldsymbol{p})_{\text {in }}=\mathscr{H}(\boldsymbol{p})_{\text {out }}=\mathscr{H}(\boldsymbol{p})$ et l'espace de Hilbert des états sortants $\mathscr{H}^{\text {ex }}$ est l'intégrale hilbertienne des $\mathscr{H}(\boldsymbol{p})$

$$
\mathscr{H}^{\mathrm{ex}}=\int_{\oplus} \mathscr{H}(\boldsymbol{p}) d^{3} p .
$$

L'espace $\mathscr{H}^{\text {ex }}$ dépend du choix de l'espace des champs de vecteurs mesurables [17]. Cet espace des champs de vecteurs mesurables se déduit de celui indiqué dans la définition de $\mathscr{H}=\int_{\oplus} \mathscr{F}(p) d^{3} p$ (cf. III) à l'aide de la transformation $U(\boldsymbol{p}, \infty)$ telle que $\mathscr{H}(\boldsymbol{p})=U(\boldsymbol{p}, \infty) \mathscr{F}$. L'application $U(\boldsymbol{p}, \infty)$ qui au champ de vecteurs $\Phi(\boldsymbol{p}) \in \mathscr{F}$ de carré intégrable fait correspondre le champ de vecteurs $U(\boldsymbol{p}, \infty) \Phi(\boldsymbol{p})$ de $\mathscr{H}(\boldsymbol{p})$ est un isomorphisme de $\mathscr{H}$ sur $\mathscr{H}^{\text {ex }}$. De plus $\mathbb{R}^{3}$ étant à base dénombrable, $\mathscr{H}^{\text {ex }}$ est à base dénombrable [12]. Il est aussi facile de voir que $\mathscr{H}\left(\boldsymbol{p}_{1}\right) \sim \mathscr{H}\left(\boldsymbol{p}_{2}\right)$ si et seulement si $\boldsymbol{p}_{1}=\boldsymbol{p}_{2}(\mathscr{F}=\mathscr{H}(\mathbf{0}))$. A chaque $\mathscr{H}(\boldsymbol{p})$ est donc associée une représentation des relations canoniques de commutation non équivalente à la représentation de Fock. 


\section{Le systéme «total»}

Si on suppose $V(x)$ borné

$$
|V(\boldsymbol{x})|<A<\infty
$$

alors $H=H^{\prime}+V$ est self adjoint. Nous allons voir que $H$ est un opérateur self adjoint pour une classe beaucoup plus large de potentiels.

a) Quelques propriétés de $H^{\prime}$ et de $H$

Dans tout ce qui suit l'indice $\varepsilon$ signifiera que l'on a remplaçé $\varrho(k)$ par

$$
\tilde{\varrho}_{\varepsilon}(\boldsymbol{k})=\left\{\begin{array}{ll}
\tilde{\varrho}(\boldsymbol{k}) & |\boldsymbol{k}|>\varepsilon>0 \\
0 & \text { ailleurs }
\end{array} .\right.
$$

Du fait que $N_{\varepsilon}(\infty)<\infty$ l'opérateur $e^{-i\left(\boldsymbol{p}, \boldsymbol{A}_{\varepsilon}(\infty)\right)}$ est un opérateur unitaire dans $\mathscr{F}$. De plus on a l'équivalence unitaire suivante:

$$
H_{\varepsilon}^{\prime} \approx H_{0}^{\mathrm{ray}}+\frac{p^{2}}{2 m_{\varepsilon}(\infty)} .
$$

En effet il est facile de verifier que

$$
e^{i B_{\varepsilon}}\left(\frac{p^{2}}{2 m}+H_{0}^{\text {ray }}+H_{I}, \varepsilon\right) e^{-i B_{\varepsilon}}=\frac{p^{2}}{2 m_{\varepsilon}(\infty)}+H_{0}^{\text {ray }} .
$$

$B_{\varepsilon}$ étant défini par

$$
B_{\varepsilon}=i \frac{e}{m} \sum_{s=1}^{2} \int \frac{d^{3} k}{k \sqrt{2 k}} \tilde{\varrho}_{\varepsilon}(\boldsymbol{k})\left(\boldsymbol{p}, \boldsymbol{e}_{s}(\boldsymbol{k})\right)\left(a_{s}(\boldsymbol{k})-a_{s}^{*}(\boldsymbol{k})\right) .
$$

D'autre part à l'aide de la représentation explicite de $e^{-i H^{\prime} t}(\boldsymbol{p})$ on peut montrer que pour $\boldsymbol{p}$ fixe et pour un ensemble dense de $\Phi$ on a la relation suivante

$$
\begin{aligned}
& \left\|e^{-i H_{\varepsilon}^{\prime} t}(\boldsymbol{p}) \Phi-e^{-i H^{\prime} t}(\boldsymbol{p}) \Phi\right\|<C(\Phi, \varepsilon)(1+|t|)^{N(\Phi)}, \\
& N(\Phi)<\infty, \quad C(\Phi, \varepsilon) \rightarrow 0 \quad \text { quand } \quad \varepsilon \rightarrow 0 .
\end{aligned}
$$

Considérons alors la représentation spectrale de l'opérateur self adjoint

$$
H^{\prime}(\boldsymbol{p})=\int \lambda d E(\lambda)
$$

et soit maintenant une fonction $f \in C_{0}^{\infty}\left(\mathbb{R}^{3}\right)$. Considérons

$$
\begin{aligned}
& E(f)=\int f(\lambda) d E(\lambda), \\
& E(f)=\int d t e^{-i H^{\prime} t}(\boldsymbol{p}) \tilde{f}(t), \quad \tilde{f} \in \mathscr{S}\left(\mathbb{R}^{3}\right) .
\end{aligned}
$$


$E(f)$ est une intégrale de Riemann forte. De (5) résulte que sur tout l'espace de Fock

$$
E(f)=s-\lim _{\varepsilon \rightarrow 0} E_{\varepsilon}(f) \quad \forall f \in C_{0}^{\infty}\left(\mathbb{R}^{3}\right) .
$$

L'équivalence unitaire entre $H_{\varepsilon}^{\prime}$ et $H_{0}^{\text {ray }}+\frac{p^{2}}{2 m_{\varepsilon}(\infty)}$ a pour conséquence puisque $H_{0}^{\text {ray }} \geqq 0$ que:

$$
E_{\varepsilon}(f)=0 \quad \text { si } \quad \operatorname{supp} f \cap\left[\frac{\boldsymbol{p}^{2}}{2 m_{\varepsilon}(\infty)},+\infty\right)=\emptyset .
$$

La propriété (6) entraine que

$$
E(f)=0 \quad \text { si } \operatorname{supp} f \cap\left[\frac{p^{2}}{2 m(\infty)},+\infty\right)=\emptyset .
$$

C'est à dire que

$$
H^{\prime}(\boldsymbol{p}) \geqq \frac{\boldsymbol{p}^{2}}{2 m(\infty)} .
$$

Le membre de droite de cette inégalité est un nombre $-c$ sur l'espace de Fock. Il en résulte que sur $\mathscr{H}=\int_{\oplus} \mathscr{F}(\boldsymbol{p}) d^{3} p$

$$
D\left(\boldsymbol{p}^{2}\right) \supset D\left(H^{\prime}\right) .
$$

$D(A)$ étant le domaine de définition de l'opérateur $A$ et $\forall \Psi \in D\left(H^{\prime}\right)$ on a

$$
\left\|p^{2} \Psi\right\| \leqq 2 m(\infty)\left\|H^{\prime} \Psi\right\| .
$$

Soit alors $V$ un potentiel de Kato $V(\cdot) \in L^{2}\left(\mathbb{R}^{3}\right)+L^{\infty}\left(\mathbb{R}^{3}\right)$ c'est à dire un potentiel tel que $\forall \alpha>0 \exists \beta<+\infty$ de sorte que

$$
\|V u\| \leqq \alpha\left\|p^{2} u\right\|+\beta\|u\|
$$

$\forall u \in D\left(p^{2}\right)$ dans $L^{2}\left(\mathbb{R}^{3}\right)$.

Mais d'après (8) on a sur $\mathscr{H}$ l'inégalité suivante

$$
\|V \Psi\| \leqq 2 \alpha m(\infty)\left\|H^{\prime} \Psi\right\|+\beta\|\Psi\|
$$

et comme $2 \alpha m(\infty)$ peut être rendu arbitrairement petit $H=H^{\prime}+V$ est self adjoint sur $D(H)=D\left(H^{\prime}\right)$ et est borné inférieurement [13]. De plus l'équivalence unitaire (4) permet de conclure que $H_{\varepsilon}^{\prime}$ a un spectre purement continu. La propriété (6) a pour conséquence immédiate que le spectre de $H^{\prime}$ est lui aussi purement continu [14].

\section{b) Etats asymptotiques du système $H$}

Soit $\Omega(t)=e^{i H t} e^{-\imath H^{\prime} t}$. Afin de montrer que l'évolution réelle du système donnée par l'hamiltonien $H$ tend asymptotiquement vers celle donnée par $H^{\prime}$ nous allons montrer que l'opérateur de Möller $\Omega(t)$ 
admet des limites fortes quand $|t| \rightarrow \infty$. Une condition suffisante pour l'existence des limites fortes $\Omega^{ \pm}$est assurée par le fait que (pour $\Omega^{+}$par exemple)

$$
\int_{t_{0}}^{+\infty}\left\|V e^{-i H^{\prime} t} \Psi\right\| d t<\infty
$$

pour un ensemble d'états denses dans $\mathscr{H}$. Considérons par exemple les états $\Psi(\boldsymbol{p}) \Phi$ de $\mathscr{H}$ definis par:

$$
\begin{aligned}
& -\Psi(\cdot) \in C_{0}^{\infty}\left(\mathbb{R}^{3}\right), \quad 0 \notin \operatorname{supp} \Psi(\cdot), \\
& -\Phi \in \widetilde{\mathscr{F}}_{0}=\bigcup_{N=0}^{\infty} P_{N} \widetilde{F}
\end{aligned}
$$

où $P_{N}$ est le projecteur qui annule les composantes d'indice plus grand que $N$.

If faut estimer la décroissance dans le temps de la norme du vecteur

$$
\left(V e^{-i H^{\prime} t} \Psi\right)(\boldsymbol{p})=\int d^{3} p^{\prime} \tilde{V}\left(\boldsymbol{p}-\boldsymbol{p}^{\prime}\right) e^{-i H^{\prime} t}\left(\boldsymbol{p}^{\prime}\right) \Psi\left(\boldsymbol{p}^{\prime}\right) \Phi
$$

pour $|t| \rightarrow \infty, \tilde{V}$ étant la transformée de Fourier du potentiel $V$. Le facteur $e^{-i} H_{0}^{\text {rayt }}$ commutant avec $V$ ne contribue pas à la norme. Il reste donc à estimer

$$
\int d^{3} p^{\prime} e^{-\frac{i \boldsymbol{p}^{\prime 2} t}{2 m(t)}} \tilde{V}\left(\boldsymbol{p}-\boldsymbol{p}^{\prime}\right) \Psi\left(\boldsymbol{p}^{\prime}\right) e^{-i\left(\boldsymbol{p}^{\prime}, \boldsymbol{A}(t)\right)} \Phi .
$$

Envisageons d'abord le cas où $t$ est positif. Pour estimer la décroissance de la norme du vecteur ci dessus on va utiliser la relation suivante:

Soit $f \in C_{0}^{\infty}\left(\mathbb{R}^{3}\right)$ on a alors:

$$
\left.\begin{array}{l}
\int d^{3} p e^{-\frac{i \boldsymbol{p}^{2} t}{2}} f(\boldsymbol{p})=4 \pi i \sum_{k=1}^{n} t^{-\left(k+\frac{1}{2}\right)} F_{2 k-1}(0)\left(\Delta^{k-1} f\right)(0) \\
-i t^{-\left(n+\frac{1}{2}\right)} \int d^{3} p \frac{F_{2 n-1}(p \sqrt{t})}{p}\left(\Delta^{n} f\right)(\boldsymbol{p})
\end{array}\right\}
$$

où $F_{n}(r)$ est l'intégrale multiple de Fresnel

avec

$$
F_{n}(r)=\int_{r}^{\infty} d r_{1} \int_{r_{1}}^{\infty} d r_{2} \ldots \int_{r_{n-1}}^{\infty} d r_{n} e^{-\frac{1}{2} r_{n}^{2}}
$$

$$
\left|F_{n}(r)\right| \leqq c_{n}(1+r)^{-n} .
$$

La relation (9) se démontre en intégrant par parties en utilisant le théorème de Green.

Dans le cas qui nous intéresse la contribution des termes tout intégrés est nulle du fait que l'origine $0 \notin \operatorname{supp} \Psi(\cdot)$. Ce que l'on utilise par contre 
est une estimation en $t$ de la norme suivante

$$
\begin{aligned}
& \left\|\frac{\partial^{k}}{\partial p_{1}^{k_{1}} \partial p_{2}^{k_{2}} \partial p_{3}^{k_{3}}} e^{-i(\boldsymbol{p}, \boldsymbol{A}(t))} \Phi\right\|_{\mathscr{F}}, \\
& k=k_{1}+k_{2}+k_{3}, \quad k_{i} \in \mathbb{Z}_{+} .
\end{aligned}
$$

Du fait que $\left[A_{i}(t), A_{k}(t)\right]=0$ on a

$$
\left\|\frac{\partial^{k}}{\partial p_{1}^{k_{1}} \partial p_{2}^{k_{2}} \partial p_{3}^{k_{3}}} e^{-i(\boldsymbol{p}, \boldsymbol{A}(t))} \Phi\right\|_{\mathscr{F}}=\left\|A_{1}^{k_{1}}(t) A_{2}(t)^{k_{2}} A_{3}(t)^{k 3} \Phi\right\|_{\mathscr{F}} .
$$

Pour majorer cette expression on peut utiliser l'inégalité suivante [15]

$$
\left\|A_{i}(t) \Phi\right\|_{\mathscr{F}} \leqq 2 N(t)(v+1)^{1 / 2}\|\Phi\|_{\mathscr{F}} .
$$

$N(t)$ étant défini par (2) et $v$ étant tel que $\Phi^{(n)}=0$ pour $n>v$. On a donc

$$
\left(A_{i}(t) \Phi\right)^{(n)}=0 \text { pour } n>v+1
$$

et

$$
\left\|A_{1}(t)^{k_{1}} A_{2}(t)^{k_{2}} A_{3}(t)^{k_{3}} \Phi\right\|_{\mathscr{F}} \leqq[2 N(t)]^{k}(v+k)^{k / 2}\|\Phi\|_{\mathscr{F}} .
$$

Utilisant les relations (2) et (9) on conclut que

$$
\left\|V e^{-i H^{\prime} t} \Psi\right\| \leqq A t^{-\left(N+\frac{1}{2}\right)}(\log t)^{N} .
$$

$A$ étant une constante et $N$ étant lié à la façon dont le potentiel décroit à l'infini. $N$ est tel que

$$
\int d^{3} x|x|^{2 M}|V(\boldsymbol{x})|^{2}<\infty \text { pour } n=0,1 \ldots N .
$$

Pour que la limite $\Omega^{+}$existe il suffit que $N=1$. En effet pour $N=1,2 \ldots$ le membre de droite de l'inégalité est une fonction intégrable de $t$ sur l'intervalle $\left[t_{0},+\infty\right)$ et il en résulte que $\Omega(t)$ tend fortement vers $\Omega^{+}$. La même démonstration peut se faire quand $t \rightarrow-\infty$. La majoration (10) montre que pour des forces à courte portée la convergence de $\Omega(t)$ vers $\Omega^{ \pm}$peut être plus rapide que n'importe quelle puissance de $1 /|t|$ sur les états que nous avons défini plus haut et qui forment un ensemble dense dans $\mathscr{H}$.

\section{Conclusion}

On a vu que $U(\boldsymbol{p}, t)$ tendait faiblement vers un opérateur $U(\boldsymbol{p}, \infty)$ qui permute les classes d'equivalence. En particulier $U(\boldsymbol{p}, \infty)$ applique l'espace de Fock $\mathscr{F}$ sur un espace de Hilbert $\mathscr{H}(\boldsymbol{p})$ auquel est associée une représentation irréductible des relations canoniques de commutation non équivalente à celle de Fock

$$
\mathscr{H}(\boldsymbol{p})=U(\boldsymbol{p}, \infty) \mathscr{F} .
$$


$U(p, \infty)$ s'interpréte physiquement comme l'opérateur qui associe à l'électron d'impulsion $\boldsymbol{p}$ son nuage formé d'un nombre infini de photons mous. On a défini $\mathscr{H}^{\text {ex }}$ par

$$
\mathscr{H}^{\mathrm{ex}}=\int_{\oplus} d^{3} p \mathscr{H}(\boldsymbol{p})
$$

l'isomorphisme entre $\mathscr{H}$ et $\mathscr{H}^{\text {ex }}$ est donné par l'application définie par

avec

$$
U(\infty)=\int_{\oplus} d^{3} p U(\boldsymbol{p}, \infty)
$$

$$
\mathscr{H}^{\mathrm{ex}}=U(\infty) \mathscr{H} .
$$

La théorie se laisse entièrement reformuler dans l'espace de Hilbert $\mathscr{H}^{\text {ex }}$. Il suffit de réinterpreter tout ce qui a été fait dans $\mathscr{H}$. Au vecteur $\Phi(\boldsymbol{p}) \in \mathscr{F}(\boldsymbol{p})$ correspond le vecteur $\hat{\Phi}(\boldsymbol{p}) \in \mathscr{H}(\boldsymbol{p})$

$$
\hat{\Phi}(\boldsymbol{p})=U(\boldsymbol{p}, \infty) \Phi(\boldsymbol{p})
$$

à l'opérateur $A$ dans $\mathscr{H}$ correspond l'opérateur $\hat{A}$ dans $\mathscr{H}^{\text {ex }}$

$$
\hat{A}=U(\infty) A U(\infty)^{-1} \text {. }
$$

Tout ce qui a été démontré sur $\mathscr{H}$ s'étend évidemment à $\mathscr{H}^{\text {ex }}$. En particulier les opérateurs

$$
\begin{aligned}
\hat{H}^{\prime} & =\frac{\hat{\boldsymbol{p}}^{2}}{2 m(\infty)}+\hat{H}_{0}^{\text {ray }}, \\
\hat{H} & =\hat{H}^{\prime}+\hat{V}
\end{aligned}
$$

sont self adjoints sur $\mathscr{H}^{\text {ex }}$ puisque les opérateurs $H^{\prime}$ et $H$ (pour des $V$ convenables) le sont sur $\mathscr{H}$. De plus l'opérateur de Möller

$$
\hat{\Omega}(t)=e^{i \hat{H} t} e^{-i \hat{H}^{\prime} t}
$$

tend fortement vers $\hat{\Omega}^{ \pm}$quand $t \rightarrow \pm \infty$.

Ainsi dans $\mathscr{H}^{\text {ex }}$ le problème de la diffusion est bien défini et les divergences infrarouges sont eliminées. La matrice $\hat{S}$ peut se définir dans $\mathscr{H}^{\text {ex }}$ par l'expression usuelle

$$
\hat{S}=\hat{\Omega}^{-} \hat{\Omega}^{+*} .
$$

C'est un plaisır pour moi de dire toute la reconnaissance que j'ai aux Professeurs R. Jost et W. Hunziker pour l'arde et les conseils quı ont permis à ce travail d'aboutir. Je remercie aussi tous mes amis de l'Institut et tout spéçialement le Dr. W. Schneider qui a lu mon manuscrit. 


\section{References}

1. Bloch, F., and A. Nordsieck: Phys. Rev. 52, 54 (1937).

2. Pauli, W., and M. Fierz: Nuovo Cimento 15, 167 (1938).

3. Friedrichs, K. O.: Mathematical aspects of the quantum theory of fields. New York: Interscience Publ. Inc. 1953.

4. Jauch, J., and F. Rohrlich: Theory of photons and electrons. Reading, Mass.: AddisonWesley 1959.

5. Magnus, W.: Commun. Pure Appl. Math. 7, 649 (1954).

6. Blanchard, P.: Travail de diplome ETH (Nov. 1965).

7. Kibble, T. W. B.: Imperial College, preprint ICTP 67/15 (April 1967).

8. von Neumann, J.: Composito Math. 6, 1 (1938).

9. Klauder, J. R., and J. Mac Kenna: J. Math. Phys. 6, 68 (1965).

10. — - and E. J. Woods: J. Math. Phys. 7, 822 (1966).

11. Streit, L.: Commun. Math. Phys. 4, 22-31 (1967).

12. Dixmier, J.: Les algébres d'opérateurs dans l'espace hilbertien. Paris: Gauthier-Villars 1957.

13. Kato, T.: Perturbation theory for linear operators. Berlin-Heidelberg-New York: Springer 1966.

14. Schroer, B.: Fortschr. Physik 11, 1-32 (1963).

15. Nelson, E.: J. Math. Phys. 5, 1190 (1964).

16. Borchers, H. J., and W. Zimmermann: Nuovo Cimento 31, 1047-1059 (1964).

17. Neumark, M. A.: Normierte Algebren, §41. Berlin: VEB Deutscher Verlag der Wissenschaften.

Ph. Blanchard

Université de Tunis

Laboratoire de Mathématiques

8 Rue de Rome (Annexe)

Tunis, Tunisie 